\title{
Requirement engineering in health care and telemedicine
}

A. Tobola, Fraunhofer Institute for Integrated Circuits, Erlangen, Germany, andreas.tobola@iis.fraunhofer.de

C. Hofmann, Fraunhofer Institute for Integrated Circuits, Erlangen, Germany, christian.hofmann@iis.fraunhofer.de C. Weigand, Fraunhofer Institute for Integrated Circuits, Erlangen, Germany, christian.weigand@iis.fraunhofer.de

\section{Introduction}

Requirement engineering (RE) covers activities involved in elicitation, analysis, specification, management and verificationof requirements.All development processesfor safety related systems begin with timeintensive requirement tasks. Especially in the medical sector RE plays a major role as all development activities refer to the previously specified unique identified requirements.Neverthelessmany projects fail due to consequences of underestimated RE activities.Smart Sensor i is a project within the "Medical Valley EMN" founded by the German ministry BMBF and industry. The project is structured in five sub projects (Smarts Sensors A to Smarts Sensors E) and one leading project on the top. The project goal is the research of a modular and scalableassistance system and the appropriate services to give peoplein care more freedom and self-determination.Taking Smarts Sensors Aas an example we show how typical RE tasks can be applied even in research and development to focus on stakeholder needs and to bring together all the synergy of a large interdisciplinary project team.

\section{Methods}

RE exists since the very early beginning of systems engineering and therefore many methods have been tested and defined very well the past decades. To finish a project in time it is essential to choose the most effective methods. In general RE starts with the stakeholder definition. A modified web-based ticket system (Trac by edgewall software) has been provided to all project members to document requirements. Those companies with direct access to stakeholders were asked to specify requirements according to previously defined methods.

\section{Results}

Stakeholder definitions, questionnaires, use case definition, wording templates, requirements analysis methods, glossaries, traceability and requirement prioritization were the most effective methods so far.

\section{Conclusion}

The work regarding RE helped us to focus on the most important things by means of the project goals. The requirements document helped to work out a detailed specification on an engineering level within a short time. Project managers were able to make important decisions regarding available resources vs. functionality based on clearly defined requirements. Overall the results of RE done this way are a basis for any activity during the projects. It provides a communication platform for engineers, economists, consulting physicians, managers and stakeholders.

${ }^{\mathrm{i}}$ More about the Smart Sensors projects: https://www.medical-valley-emn.de/taxonomy/term/246 